\title{
Does a Narrow Definition of Medical Professionalism lead to Systemic Bias and Differential Outcomes?
}

\author{
A Narrative Commentary
}

\begin{abstract}
The concept of medical professionalism is enshrined in the principles of Good Medical Practice (GMP), upheld by the General Medical Council (GMC), and is applicable to all doctors as well as undergraduate students in the United Kingdom. The principles were conceived, developed and implemented to ensure that the highest standards of medical practice are adhered to for the safety of the public and to retain trust in the medical profession. The GMC has a statutory duty to ensure that professional standards are maintained. Approximately $1 \%$ of doctors on the medical register are subject to investigation by the GMC, based on referrals made by employing organisations or the public.
\end{abstract}

While appropriate GMC investigation is essential to maintain standards, patient safety and public confidence, there are inherent inequalities in the process resulting in widely different outcomes for certain groups of students or doctors based on Black and minority ethnicity, male gender and non-UK primary medical qualification often leading to devastating consequences.

This narrative review considers the contributors to differential attainment (DA) and the impact on the health service, patients and individual wellbeing. It explores the tenets of a current narrow definition of professionalism which, while representing the unidimensional White majority view, ignores the huge diversity of the workforce and the public, thus exposing significant tensions for groups of professionals. The Covid-19 pandemic has exposed the inherent systemic bias in the health service for both professionals and the public. This review recommends root and branch reform of the definition of professionalism, by engagement with the public and the workforce, to incorporate principles of equality, diversity and inclusion, which the authors believe will create the environment for a just and equitable professional experience. ${ }^{1}$

\section{Keywords}

Differential attainment; medical professionalism; BME; GMC; Regulation
Ananta Dave, Naureen Bhatti, Rosanna Geddes, Triya A Chakravorty, Satheesh Mathew, Joydeep Grover, Shivani Sharma, JS Bamrah \& Indranil Chakravorty BAPIO Institute for Health Research, Bedford, UK

\section{Ananta.dave@nhs.net}

Cite as:

Dave, A., Bhatti, N., Grover, J., Geddes, R., Chakravorty, T., Mathew, S., Sharma, S., Bamrah, J., \& Chakravorty, I. (2021).

Does a Narrow Definition of Medical Professionalism lead to Systemic Bias and Differential Outcomes? : A Narrative Commentary. Sushruta Journal of Health Policy \& Opinion, 14(1), 1-12. https://doi.org/10.38192/14.1.13

Article Information

$\begin{array}{ll}\text { Submitted } & 13.2 .21 \\ \text { Revised } & 20.2 .21 \\ \text { ePub } & 23.2 .21\end{array}$

ePub

23.2 .21

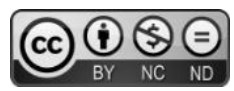




\begin{abstract}
Introduction
Differential attainment (DA) or outcomes in the medical profession describes the gap in achievement or outcomes between cohorts of doctors based on factors other than their individual actions, ability or academic prowess and is a manifestation of inherent systemic bias or inequalities. ${ }^{2,3}$ The evidence to date overwhelmingly demonstrates that within the entire spectrum of the medical profession race, ethnicity, gender, disability and socio-economic factors are prime determinants of DA. Particularly that Black and Minority Ethnic (BME) groups are disadvantaged compared to their White counterparts ${ }^{4}$, those who trained and qualified overseas and women in career progression as well as pay 5 . The causes of DA are complex, multifactorial and relate to issues which may be dating back into history and legacy. However, there are associations observed about doctors' gender, countries of origin, primary medical qualifications and ethnicity among others.
\end{abstract}

\section{General Medical Council \& Public Scrutiny}

In the United Kingdom, the responsibility for regulation and upholding medical professionalism rests with the General Medical Council (GMC) currently constituted under the Medical Act of 1978, which was legislated following the Merrison Committee's report and led to restructuring of disciplinary processes amongst other reforms. ${ }^{6}$ The Good Medical Practice guidelines (GMP) were first published by the GMC in 1995 and has been continually revised since 7 . The GMP defines the set of standards that doctors need to adhere to and is essential for maintaining public trust and confidence. Due to a series of high profile instances of catastrophic patient outcomes as a result of poor institutional governance, lack of managerial oversight, medical error or criminal negligence and incompetent leadership, the trust in the profession's ability to self-regulate had been seriously compromised (such as the Bristol heart scandal ${ }^{8}$ or the Shipman serial killings ${ }^{9}$ ). In the Shipman Inquiry report, Dame Janet Smith reiterated that the system was not working as well as it should, that there was much dissatisfaction with the existing arrangements, which were fragmented, not sufficiently professional, applied to very variable standards in different parts of the country and did not meet the needs of the public. Thus in 2003, there was a major reform of the GMC, when membership of the council was reduced from 104 members to 35 and the proportion of lay members rose to $40 \%$ and then to $50 \%$ lay members. ${ }^{10}$ The election of medical members by the profession was replaced with appointment by the Privy Council. The requirement for revalidation was introduced in 2012, the GMP underwent a major revision in 2013 and subsequently the licence to practice requirements were implemented. ${ }^{7}$ These were developed when race/ethnicity considerations were non-existent or minimal at best.

In 2017, the UK government initiated a sweeping consultation for reform of regulation and professional standards. ${ }^{11}$ The justification for reform was articulated as the responsibility of the regulator as not only oversee the professionalism of every individual practitioner, but as the guardians of the ethos and culture of the profession as a whole. As the profession needs to adapt to the opportunities and challenges of the economic, demographic, technological and epidemiological advances of the coming decades, it was felt to be vital that the regulators were able to respond to these changes. They must be able to lead the adaptation of professional standards to the changing realities of ensuring safe, effective and respectful clinical care in a way that is efficient, effective and affordable. The Marmot report ${ }^{12}$, the Black lives matter movement ${ }^{13}$ and the disparities unearthed in mortality or outcomes with the COVID-19 pandemic ${ }^{1,14}$ has exposed the deep seated, inherent bias and inequalities in the healthcare system in the UK, and globally. It is therefore imperative that we undertake an honest and deep examination of the equality, diversity and inclusion impact of the concept of medical professionalism, engage a whole system contribution ${ }^{15,16}$ to the reform of the regulation, and the regulator, as well as take bold measures to achieve a just and fair system for all within the medical profession thus delivering the best outcome for patients.

\section{Impact on Health \& Well-being}

The result of disproportionate referrals and disciplinary processes as well as the perceived discriminatory regulatory processes can have a significant effect on a doctor's health and career. Indeed, referral to the regulator has been identified as one of the factors increasing risk of suicide in doctors $^{17}$. Recognising and addressing structural inequalities is important to ensure fairness in regulatory processes and hence trust in the regulators and the profession, as well as morale, wellbeing and retention of doctors.

\section{Patient Safety}

Evidence from a number of inquiries into patient safety breaches in the National Health Service (NHS) has demonstrated the catastrophic consequences of poor regulation, inadequate scrutiny of medical practice and lack of robust governance systems. ${ }^{9}$ While the symptoms of failure are often clinical in nature, such as poor standards of care, avoidable mortality and morbidity, distressed patients and their families, the pathology of failure is usually 
organisational, concerned with things such as organisational leadership, management structures and systems, organisational culture, interprofessional relationships and teamwork. ${ }^{18}$ The Francis Inquiry in 2013 , examined the causes of the failings in care at Mid-Staffordshire NHS Foundation Trust between 2005 and 2009. The report made 290 recommendations, including: openness, transparency and candour throughout the health care system (including a statutory duty of candour), fundamental standards for health care providers and improved support for compassionate caring, committed care and stronger healthcare leadership. There have been several inquiries and recommendations since requiring NHS organisations, the regulator and the UK government to improve standards of governance, protect those raising concerns, provide adequate support and training of staff, ensure workforce numbers including culturally tailored induction as well as proficiency in the English language. ${ }^{19}$ While it is undisputed that, competent and effectively regulated doctors are crucial to delivering safe and good quality patient care, there is a real imperative for getting regulatory and disciplinary processes right and develop mechanisms that identify concerns at a much earlier stage, long before they have reached the stage of referral to the GMC for a Fitness to Practise (FtP) concern.

\section{Aim}

In this review, we look at DA in the context of medical professionalism, exploring specifically, the impact of decisions and judgments made about a doctor's professional practice by employers and regulators when concerns have been raised about their FtP. This is an important area to consider given the major personal, organisational and patient safety implications of rehabilitative or punitive action. Our aim is also to interrogate the evidence on the circumstances in which such referrals and actions are more likely, and to critically discuss the inherent biases within this process.

\section{Methods}

We have undertaken a narrative review of the published literature and reports focussing on the concept of professionalism and framing them in relation to the GMC domains of Good Medical Practice $^{7}$ which form the basis of regular appraisals as well as investigation of a doctor's practice, if there are concerns. Our review has considered the evidence for factors contributing to differential outcomes in regulatory processes. While we have applied a broad lens that is inclusive of all protected characteristics under the Equality Act, the available data and narrative analysis are centred on race and ethnicity.

\section{Discussion of Findings}

\section{Medical Professionalism}

The Merrison Committee's report in 1975 was quite candid in not attempting to solve all the problems of regulating the medical profession, but primarily to recommend machinery for the solution of problems and in some areas to point the direction of possible solutions which the profession itself must work out. The report suggested a framework within which difficulties could be resolved satisfying the profession and the community it served. It envisaged this to be sufficiently efficient and flexible to take account of rapid continuing progress in science and technology, the changing use of medical resources, and the movement in attitude and outlook of the profession and public alike. ${ }^{6}$ When the NHS was set up an implicit 'compact' was made between doctors, patients and society. ${ }^{20}$ The government guaranteed free access to healthcare, the public accepted their obligation to fund this through taxation and doctors were given significant clinical freedom and minimal accountability as 'professionals'. ${ }^{21}$ The problem with this social compact of professional autonomy has been that not only did the profession as a whole assume autonomy for itself, but so did each practitioner. The traditional professional image was of the selfless clinician, motivated by a strong service ethos, with unique skills and knowledge, working all hours to restore the health of their patients. This is reflected in the GMC definition of the "good doctor" as one who makes "the care of their patients their first concern: they are competent, keep their knowledge and skills up to date, establish and maintain good relationships with patients and colleagues, are honest and trustworthy, and act with integrity and within the law"7. Good medical practice describes what is expected of all doctors registered with the GMC, and in order to maintain their license to practise, they must demonstrate, through the appraisal and revalidation process, that they work in line with the principles and values set out. However, there has been no explicit attempt to embed the professional standards through public engagement via the lens of a changing society, a diverse society by the law makers and the GMC and therefore no responsibility for taking into account the awareness of a systemic bias and inherent unfairness. The unidimensional interpretation of professionalism standards thus provides inherent privileges of Whiteness and discriminates against non-Western and non-White professionalism standards such as related to dress code, speech, work style, and timeliness and thus created a significant disparity on who was held accountable for 'violations' of such standards. Therefore, one may argue that the $45 \%$ of medical staff in the UK NHS are inherently at a 
systemic disadvantage and at higher risk of falling foul of such narrow definition of professional standards.

The concept of the medical professional has been explained through other paradigms too such as the frequently cited CanMEDS framework developed in Canada by the Royal College of Physicians and Surgeons ${ }^{22}$. This defines the competent physician as a medical expert needing to demonstrate abilities in as a communicator, collaborator, leader, health advocate, scholar and professional, which approximately map onto the domains of $\mathrm{GMP}^{7}$. The last two decades has brought a broader exposition of the principles of good practice and conduct. In so doing an obligation has been placed on clinicians to meet more explicit and higher professional standards. A statutory duty of quality placed on all NHS organisations in the late 1990s is being implemented through the concept of clinical governance. All these changes have highlighted the duty of clinicians to continuously strive to develop professionally-to acquire and retain clinical skills, to access and use best evidence, to participate in planning for quality, and to evaluate and optimise processes of care. New and explicit forms of accountability have been required and captured in the concept of clinical governance. This new accountability has redefined the relationship of doctors with the public, with their patients, and with their employer. ${ }^{23}$

It is recognised that healthcare organisations that value equality and diversity of their workforce, also benefit by their staff emulating a culture that values diversity and person-centred approaches to care. This is to promote patient dignity. ${ }^{24}$ However, there has been no explicit attempt to embed the professional standards through public engagement via the lens of a changing society, a diverse society, and taking into account the awareness of a systemic bias and inherent unfairness. These escalating requirements and unidimensional models of professionalism put high expectations on a doctor, which can feel burdensome, and may affect the way that professionalism is assessed, especially in doctors trained overseas and from diverse cultures.

\section{Conflation of Prevalent Culture \& Professionalism}

Within widely accepted definitions of professionalism there is conflation of professionalism with 'being proper'. American grassroots organisers-scholars Tema Okun and Keith Jones assert that standards of professionalism are heavily defined by systemic, institutionalised centring of White supremacy culture ${ }^{25}$. In the workplace, this both explicitly and implicitly privileges Whiteness and discriminates against non-
Western and non-White professionalism standards related to dress code, speech, work style, and timeliness. There is an expectation to change to conform to these standards to make others feel comfortable. The challenge of "race-conscious professionalism" is often used to understand the dual obligation encountered by many minority ethnic physicians not only to maintain excellence in their profession but also to leverage their professional stature to improve the well-being of their communities. Often a desire to have their work focused on the community or being sensitive to the diverse ways of the community may be at odds with prevalent expectations or the culturally insensitive, narrow definition of GMP standards. 26

Definitions of professionalism underpin the trust the public has in doctors. While doctors remain highly trusted in all public polls, social and political factors, together with the achievement and promise of medical science, have reshaped the expectations of the public and the attitudes of employers and regulators. A number of high-profile incidents such as the Bristol Heart Scandal ${ }^{8,18}$ and the Harold Shipman case ${ }^{9}$ have further challenged the right of doctors to be the main arbiters of professionalism and led to widespread changes in regulation. The Francis report ${ }^{19}$ into the Mid-Staffordshire scandal, where administrative targets led to the development of a toxic management style and significant detriment to patients, called for the medical profession to review its values and reevaluate what the core principals of professionalism were. On the contrary, the break of trust with the government during the junior doctor contract negotiations ${ }^{27}$, blame culture manifest in the BawaGarba case ${ }^{28,29}$ and other such instances have dented the confidence of medical students and junior doctors in the fairness and responsiveness of the regulation regime.

\section{Role Models \& Bias}

Damaging incidents within the profession were accompanied by important societal transformation, driven by the millennial generation of young doctors demanding changes in the way they work and moving away from medicine as a vocation, to medicine as a job ${ }^{30}$. Increasing diversity in the contemporary workforce means that young doctors no longer see the older - predominantly White male doctor- as their role model. Indeed, role modelling has the potential to reinforce unconscious bias if we as educators see it as the way to 'teach' professionalism.

The Fenton Review into mortality associated with Covid-19 found that it has disproportionately affected those from BME backgrounds, those who were born outside of UK and Ireland, highlighting 
racism, stigma and distrust as underlying the existing inequalities 31 . Furthermore, while $44 \%$ of medical staff are BME, $95 \%$ of doctors who died were BME, with British Medical Association (BMA) and British Association of Physicians of Indian Origin (BAPIO) surveys consistently finding concerning disparities in doctors' experiences by ethnicity ${ }^{32}$. Doctors from BME backgrounds reported feeling less confident that appropriate adjustments had been made to mitigate risk, felt less confident about Personal Protective Equipment (PPE) provision and feeling safe to report PPE shortages, and reported higher rates of bullying and harassment during the pandemic period ${ }^{32}$. The relevance of these issues is the exposure of wider systemic injustices of discrimination, which is under-recognised in the literature on professional identify within medicine. ${ }^{33}$

\section{Differential Attainment}

There are strong indications from the literature that outcomes regarding professional competence and conduct may demonstrate bias and association with factors beyond an individuals' control. According to the GMC, $1.1 \%$ of BME doctors were referred to them by employers between 2012-17 compared to 0.5\% of White doctors ${ }^{34}$. Data published by the Workforce Race Equality Standard (WRES) which requires organisations to demonstrate progress against nine equality indicators, shows consistent differences in appointment to leadership positions, experiences and outcomes between BME staff and their White counterparts (WRES indicators are measured only in secondary care i.e. hospitals in England and apply to all healthcare staff not just doctors) ${ }^{35}$. It is unlawful to discriminate on the grounds of protected characteristics such as ethnicity, gender, age, maternity, sexual orientation and disability, as detailed in the Equality Act of 201036. However, as per the GMC's 'Fair to Refer' report employers and healthcare providers are more likely to refer doctors who obtained their Primary Medical Qualification (PMQ) outside the UK and those who are from BME backgrounds than they are to refer UK qualified or White peers. ${ }^{34}$ Women were much less likely to be referred to the regulator 37,38 . Complaints or referrals from employers are more likely to result in an investigation being opened, more likely to result in a sanction being applied, than complaints from other sources $^{34}$. For instance referrals from employers make up only $4.3 \%$ of all referrals, but $77 \%$ of these result in a GMC investigation, compared with only $9 \%$ of complaints from patients and the public.

In 2016, 26\% of the doctors registered with the GMC, qualified from outside the European Economic Area $(E E A)^{39}$. Doctors perceived as outsiders (such as doctors trained outside the UK) are not given the support they need by supervisors and colleagues and are more likely to end up being blamed and facing disciplinary action when things go wrong ${ }^{40,41}$. There are many reasons for this including poor induction, support, and feedback, which is particularly applicable to International Medical Graduates (IMGs) ${ }^{43}$ who are also more likely to receive serious rulings at all levels of the GMC's fitness to practise procedure, than are UK trained doctors. ${ }^{44} \mathrm{~A}$ retrospective study (commissioned by the GMC) found that doctors who qualified in the European Union or further afield were more likely to be referred for investigation at the initial triage of their fitness to practise case, to be sent for adjudication after assessment at the investigation stage, and to be erased or suspended from the medical register if their case went to a hearing. Doctors from BME backgrounds and IMGs do not think that the GMC treats all doctors fairly. ${ }^{45}$

However, in a retrospective review of 187 fitness to practise cases $^{46}$, decisions were deemed to be appropriate, and complying in line with the GMC's published criteria. Thereby demonstrating no real evidence of bias or discriminatory practice in most of the cases. However in some of the cases, the decisions made were not fully reasoned and the review was hampered by deficiencies in the data recording and decision-making processes which made it impossible to tell to what extent racial bias existed.

As demonstrated in the GMC's Fair to Refer report, referrals from employers, healthcare providers and Persons Acting in a Public Capacity are far more likely than complaints from the public to be investigated by the GMC (84\% compared with 16\%) and to subsequently lead to sanction. Doctors from BME backgrounds particularly those with their PMQ (Primary Medical Qualifications) from outside the UK are more than twice as likely to be referred to the GMC as their White counterparts. Furthermore, IMG and BME doctors have been concerned that sanctions issued against them by the GMC are harsher than the sanctions issued to their White colleagues for similar mistakes. The GMC believes (based on independent reviews) that there is no explicit bias in its decision making ${ }^{16}$, as personal characteristics or place of PMQ were unrelated to the seriousness of regulatory outcomes. Instead, engagement (attendance and legal representation), allegation type, and referral source were importantly associated to outcomes ${ }^{17}$.

The authors have been unable to obtain data from the GMC on outcomes of concerns raised about doctors based on ethnicity to perform an independent analysis, therefore it is difficult to verify such claims raised by the GMC. Similarly, it has not been possible to get data from Universities by 
protected characteristics on outcomes for medical students referred for concerns about performance or conduct.

\section{DA in Undergraduate Medical Education}

The attainment gap between BME doctors and their White peers is seen at all stages of the medical profession, including at an undergraduate level 3,4 . Therefore, it is important to consider the potential long-term impacts that medical school experiences may have on a doctor's career. Issues such as discrimination and differential attainment faced in medical school have the potential to affect career progression. This is why understanding the problem at an undergraduate level is so important, especially given that BME students make up forty percent of all medical undergraduates ${ }^{48}$. Referring medical students to fitness to practice procedures involves the University authorities rather than through the GMC process, hence data regarding undergraduate level referrals is not readily available. Nevertheless, there is evidence to suggest that BME medical students face issues such as discrimination and harassment that may impact their wellbeing, professionalism and potentially future referrals ${ }^{49}$.

There are several factors that may contribute to the negative experiences of BME medical students, including microaggressions, undermining and racial harassment in both the university campus as well as on clinical placements ${ }^{50}$. The issue with racial discrimination is one that has been present in medical schools for several decades. A report in 1988 highlighted the racial discrimination in St George's medical school applications ${ }^{51,} 52$. Facing racial harassment is distressing at any level, and is thought to contribute to differential attainment seen in medical students ${ }^{53}$. Other factors may include lack of mentorship and role models.

In an inquiry into racial harassment in UK universities, the Equality and Human Rights Commission (EHRC) found that around a quarter of BME students had experienced racial harassment at university ${ }^{54}$. Furthermore, it is believed that racial harassment may be underreported within medical schools, due to students' reluctance to come forward. For example, a survey conducted by the BMA in 2018 found that BME medical students were more likely to report being afraid to speak up about harassment compared to their White peers ${ }^{55}$. There are many potential reasons for the underreporting of racial harassment. These include concerns that students' complaints will not be taken seriously, fear of being labelled a "trouble-maker" and lack of information about how to report incidents, especially in the context of clinical placements ${ }^{56}$. It is for these reasons that the BMA launched a charter in 2020 for medical schools which aimed to prevent and effectively deal with racist incidents. It gives guidance for students to deal with and prevent racial harassment, which includes advice for medical schools on how to create robust procedures for handling complaints, as well as integrating Equality, Diversity and Inclusion (EDI) training into the mainstream curriculum. It is clear that action needs to be taken to make BME medical students feel safe and supported in their learning environment.

Another potential contributing factor to the problem is the lack of diversity on the medical school teaching staff. A BMJ report found that although $40 \%$ of undergraduates were BME, only $13 \%$ of the teaching staff were ${ }^{50}$. This could lead to a disconnect in learning and teaching methods, which may impact on exam performance. Furthermore, the lack of positive role models and mentors from similar backgrounds may demoralise BME students.

\section{Fitness to Practice Investigative Process}

The authors considered the process involved in a doctor being referred to the GMC and possible outcomes. The GMC has a statutory duty to quality assure the UK medical workforce by two mechanisms. Revalidation requires all doctors to demonstrate on a regular basis that they are up to date and fit to practise in their chosen field and able to provide a good level of care ${ }^{57}$. The fitness to practice procedures are invoked following a complaint about a doctor that raises a concern about their professional practice. When the complaints are deemed sufficiently serious by the GMC's case examiners, they are referred to the Medical Practitioners Tribunal Service (MPTS). Although funded by the GMC, the MPTS acts independently of it and reports to Parliament. The MPTS will decide whether or not to impose a sanction, which include, in decreasing order of severity, the doctor being erased or suspended from the medical register, having conditions imposed on their registration, a doctor agreeing to undertakings or a doctor being given a warning. We refer to these sanctions collectively as ESCUW (Erasure, Suspension, Conditions, Undertakings or Warnings ${ }^{58}$. The GMC FtP procedures are governed by the Medical Act 1983 and the GMC (Fitness to Practice) Rules 2004, under which a doctor's fitness to practice can be impaired due to misconduct, deficient performance, a criminal conviction or caution, adverse physical or mental health, determination by regulatory bodies in the British Isles or overseas or not having the necessary knowledge of English ${ }^{59}$. 
Table 1: Illustrative Case Studies from the files of Medical Defence Shield demonstrating anecdotes of potential disproportionate outcomes

Index case - Dr XY was a consultant in
intensive care medicine and
anaesthesia
the Register as it had been found Tribunal (MPT) that they had accepted work whilst on sick leave.

\section{Case B}

Dr CD's name had been erased in 2018

as they had wrongly written a prescription for a family friend.
Dr AB's name had been erased from proved at Medical Professional
The Dr was suspended for having been on call for NHS and private hospitals concurrently on numerous occasions (over 30)-misconduct that had potentially endangered patients.
Dr XY a White doctor had their 12month suspension cut short after their NHS trust wrote to the GMC in response to their guidance * saying that they were needed to help fight the covid-19 pandemic.

At the time of application, they had been erased for 4 years and 3 months, with only 9 months before being able to apply for restoration to the register.

Dr AB supplied substantive logs and audits of their work and actions whilst erased, letters of support from colleagues, family and friends, as well as showing their dedication to the medical profession through voluntary work. Their application was refused.

The GMC had originally only sought suspension at the MPT

Alongside their application, Dr CD provided multiple letters of support, references, evidence of courses and Continuing Professional Development (CPD) and testimonials, including one from the Practitioner Health Program recommending their restoration. Their application was refused.

Case C
Dr EF's name was erased in 2018 due
to a patient complaint which had been
made in another country.

They had been maintaining their clinical knowledge and skills through CPD as well as teaching and lecturing other medical professionals and volunteering. Interestingly they are still allowed to practice in the country where the complaint was made, after having being investigated by the local medical council.

Case D

Their request for early review of the suspension was refused by the GMC and they were informed they would have to wait another 2 months until the Interim Outcomes Tribunal (IOT) review.
Their application was refused.

Their suspension was then revoked at IOT, 2 months later, unnecessarily delaying their return to help throughout the pandemic.

misconduct not relating to their clinical skills and that did not endanger patients.

Further to the GMC guidance*, four BME doctors (their cases described briefly Cases A-D) applied to have their sanctions relaxed and/ or revoked so they could support and work for their NHS Trusts during the pandemic. None of them were successful in their application. Cases A to D were supported by extensive logs, recommendations and detailed insightful personal pleadings from the doctors themselves, with the help of defence organisations. The index case refers to a White doctor who's suspension was revo ked appropriately by the GMC.

\section{Whistleblowing - Case Illustration of Dr Raj Mattu}

One of the most high-profile NHS whistle-blower case, Dr Mattu a Consultant Cardiologist was subjected to a 12-year 'witch hunt' by University Hospital of Coventry and Warwickshire NHS Trust due to raising concerns publicly in a BBC interview about medical negligence arising from poor facilities at his hospital. After launching disciplinary action, the Trust was found to have conducted a campaign of bullying and intimidation to force his resignation. After the Tribunal reversed the suspension and instructed the Trust to provide retraining, the Trust then downgraded the level of reskilling they were compelled to provide, thereby preventing Dr Mattu from returning to his original senior position in the hospital. The NHS Trust reported over 200 false allegations against $\mathrm{Dr}$ Mattu to the GMC between 2002 and 2013. The GMC began to investigate these allegations through the police before eventually rejecting all the false claims made by the Trust and closing his case with no further action. This is an illustration of the employer referring a doctor to the GMC over a prolonged period and subjecting them to unfair treatment, bullying and harassment as a response to his raising concerns about patient safety. The causes behind this type of action from the employer remain unclear but the impact of this discrimination on Dr Mattu's health and wellbeing and indeed on other doctors who are subjected to bullying, harassment and unfair complaints cannot be under-estimated.

\section{Wellbeing and Professionalism}

Wellbeing encompasses people's satisfaction with their life, career and future. In medicine, wellbeing is increasingly acknowledged as something that has tangible impacts on healthcare services, via patient safety, patient satisfaction and workforce retention ${ }^{62}$. When compared to the general population, doctors are at a higher risk of stress, anxiety, depression, substance abuse and suicide ${ }^{63,64}$. There are several potential reasons, including long working hours in understaffed and 
under-resourced environments, high-pressure scenarios and the risk of referral to regulators or face litigation. Wellbeing is not only important for doctors themselves, but for patients too. There is a wealth of evidence to suggest that workplace stress in the healthcare sector affects the quality of care of patients ${ }^{65}$. For example, a study conducted in the United States of America (USA) showed that doctors with high levels of burnout had a higher risk of making a major medical error than those who were not burned out ${ }^{66}$. This shows how wellbeing can impact doctors' fitness to practise.

Doctors from BME backgrounds find their wellbeing more threatened than their White peers. For example, they experience higher levels of discrimination at work ${ }^{35}$, which is known to have significant impact on physical health and stress. Such scrutiny takes a serious toll, it severely impacts careers and has led to several cases of suicide. From 2005 to 2013, twenty-eight doctors under investigation from the GMC took their own lives $^{17}$. Of this cohort, $71 \%$ were male, however data regarding their ethnicity was not published. The full impact of wellbeing on medical practice is beyond the scope of this document. Nevertheless, it is important to appreciate how wellbeing impacts on professionalism. Doctors need the support of their peers and their organisations in order for them to better care for themselves and their patients. Evidence suggests that an effective strategy to enhance wellbeing involves both individual targeted intervention such as mindfulness and other approaches, as well as organisational interventions such as reducing workload and enhancing teamwork 67,68 . Intervening early is crucial in preventing doctors' health from worsening and developing mental and physical illnesses.

\section{Conclusions}

Our analysis shows that doctors who obtained their primary medical qualification outside the UK and those who are from a BME background are more likely to have formal disciplinary investigations and be referred to the GMC with concerns about FtP by their employers and healthcare providers, compared to their UK qualified BME or White peers (the last group least likely to be referred). ${ }^{34}$ The GMC is aware that employers make up the majority of the referrals compared to complaints from the public $(84 \%$ versus 16\%). Our analysis has also shown that being male, aged over 50 years and being from a BME background are factors that contribute to a disproportionately higher likelihood of formal disciplinary processes being undertaken.
Furthermore, doctors from BME backgrounds and IMGs are more likely to receive harsher outcomes compared to their White or UK qualified peers.

Therefore, although there are possible contributors to differential outcomes of regulatory processes such as isolation, poor supervision, inadequate induction and lack of timely as well as meaningful feedback, there is clear evidence of systemic bias in the way that breaches to professionalism standards are implemented. There is also evidence that implementation of professionalism standards may implicitly privilege 'Whiteness' and discriminate against non-Western and non-White professionalism standards. While we present below some recommendations to take forward, we are aware that there is a lack of granular data regarding employer disciplinary processes and GMC referrals and investigations regulatory processes either in the public domain or in a readily accessible way, which will help us to delve deeper into the differential outcomes of regulatory and disciplinary processes.

\section{Recommendations}

\section{Open Discourse on Reforming Professional standards}

Much of the defined standards of professionalism were designed without consideration of race and gender equality and amplify established White stereotypes. These discriminators, while small at each step, serially compound detriment which becomes severe by the time a doctor's professionalism is challenged by the GMC. Unless there is a root and branch analysis and redesign of standards used to define professionalism, starting from secondary schools, entry into medical schools, medical education, training, employer process and regulatory reform, any steps taken will only paper over the cracks. We must realise that the current definition and implementation of professionalism itself is a significant contributor to the adverse outcomes faced by non-White doctors. It can be argued that this discrimination exists in the wider society, but that cannot be an excuse. Healthcare could an exemplar in leading the way to help solve this vexatious problem. With the ongoing government initiated consultation on the new regulation standards for all healthcare professions, the imminent new reform of the NHS, health and social care, the time is ripe now to engage actively with the whole spectrum of big society, to promote a wide ranging, culturally diverse understanding of medical professionalism. This work should be led by the NHS Race and Health Observatory and 
engage all representative organisations from the professions and the public.

\section{Medical Workforce Race Equality Standards}

The newly introduced Medical Workforce Race Equality Standard (MWRES) will add the granularity required to ascertain a better understanding of race disparities in the medical workforce. Of the eleven MWRES indicators, four reflect variation in career progression and pay, six represent medical staff perceptions of how they are treated by colleagues, employing organisations and patients, and one highlights the diversity of the councils and boards of medical institutions. It is a welcome start to the process of monitoring differential attainment and experience of NHS doctors and translating evidence into appropriate practice and policy to breakdown the structural barriers to race equality ${ }^{69}$. We recommend that the pilot is evaluated as soon as possible and the MWRES be rolled out across all relevant organisations, including primary care.

\section{Transparency of Data}

Collecting good quality data on ethnicity and other protected characteristics when medical professionals are referred for disciplinary or regulatory processes from medical school, through training and post qualifying is essential. The GMC, NHS Trusts and HEE must work together to identify the indicators and outcome measures required at employer level as at the level of GMC. This will enable us to understand the reasons for example, why doctors from BME backgrounds are referred at twice the rate to the GMC. It will also enable us to evaluate the effectiveness of interventions implemented. The GMC should proactively share this data appropriately anonymised with all MDs and ROs. There must be external validity of this data and accountability for organisations which fail to achieve the expected benchmarks.

\section{Embedding Cultural Diversity}

Differences in cultural understanding of professionalism, and in communication styles and use of language seem to determine the degree of trust and confidence in the doctor. It is essential that professionalism is understood from a multicultural perspective so that doctors who are not from the dominant culture do not become unduly disadvantaged. While biases and prejudices may not be completely eliminated, a robust system of checks and balances, and regular review of the processes and outcomes can minimise bias and increase equity. There is also a need for informing and educating the public regarding the value of ethnic and cultural diversity in the medical workforce.

\section{4a. Avoidance of Othering}

The GMC Fair to Refer report also says: "In groups and out groups exist in medicine including relating to qualifications (including by country and within the UK by medical school) and ethnicity (including within BME populations)." Members of ingroups can receive favourable treatment and those in out groups are at risk of bias and stereotyping. These institutional discriminatory cultures have to be dismantled if we are to see a just culture.

\section{4b. Avoidance of Stereotyping \& Micro-aggressions}

As the diversity of the workforce increases while we continue to define professionalism through a narrow cultural lens doctors from ethnic minority backgrounds continue to face disadvantage throughout their careers. Despite being selected for high academic achievement these doctors experience worse outcomes during recruitment for foundation, specialty training, and consultant posts; are more likely to fail examinations; and progress more slowly through training. This continues throughout careers with BME doctors reporting repeated micro-aggressions and stereotypical assumptions. Their concerns are minimized or dismissed unless backed by a White colleague and they continue to be marginalised throughout their careers.

\section{4c. Embedding a culture of anti-racism beginning at medical school}

Work towards an anti-racism culture from the beginning of medical careers, at medical schools and embed this throughout all the institutions where doctors, learn, train and provide medical care.

\section{Accountability}

It is crucial that the GMC works with NHS Trusts and arm's length bodies to ensure processes, including decisions to refer to the regulator, are fair, non-discriminatory and culturally sensitive, and supported by data that is open to appropriate external scrutiny. It is also essential for the GMC to implement previous recommendations from high profile cases and inquiries.

\section{5a. Responsible Officer Advisory Groups}

Referrals made by employers to the GMC are through the Medical Director (MD) and 
Responsible Officer (R0), who are often the same person in many Trusts. Strengthening and making the decision making process fairer at this stage can make a crucial difference in the way concerns are dealt with, and ultimately referred to the GMC. Life altering implications for a doctor's career and life need to be made with all due care, supported by the right information, values of equity, compassion and openness, and robust processes. An appropriate way of ensuring that a fair, well considered decision is made is to have a group of people who work closely with the MD in arriving at the right decision at the employer level regarding investigating concerns. Some Trusts have set up a ROAG (Responsible Office Advisory Group) led by the MD/RO with appropriate representation from doctors, Human Resources staff, EDI leads, Nonexecutive Directors, lay representation and patient or carer representatives as appropriate who work together to assess whether a GMC referral is necessary and appropriate. We recommend that all Trusts consider this approach and review its effectiveness regularly.

\section{5b. Diversity in Senior Leadership}

It is also crucial, if we are to embed fair, equitable cultures in organisations, that we have representative and diverse Board level leadership. In 2019, 19.7\% of staff working for NHS trusts and clinical commissioning groups (CCGs) in England were from a BME background; this has been increasing over time. In contrast, $8.4 \%$ of board members in NHS trusts were from a BME background. As $44 \%$ of doctors in the NHS are from the BAME background, a lot of work remains to be done in this area to bridge the gap.

\section{Culturally Competent Induction}

The emphasis must on preparing and supporting IMGs to understand professional practice and cultural contexts in the UK. To that effect, a robust and comprehensive induction programme by the

References

1. NHS England » Action required to tackle health inequalities in latest phase of COVID-19 response and recovery

https://www.england.nhs.uk/about/equality/equalityhub/action-required-to-tackle-health-inequalities-inlatest-phase-of-covid-19-response-and-recovery/

2. Chakravorty, I., Daga, S., Chakravorty, S., Bamrah, J., \& Mehta, R. (2020). Protocol for Thematic Synthesis of Differential Attainment in the Medical Profession 'Bridging the Gap' Series: Alliance for Equality in Health Professions. Sushruta Journal of Health Policy \& Opinion, 13(3). https://doi.org/10.38192/13.3.17

3. Nunn, S. Understanding differential attainment across medical training pathways: A rapid review of the literature. 89. https://www.gmc-uk.org//media/documents/gmc-understanding-differentialattainment_pdf-63533431.pdf
GMC as well as the employer where IMGs first start work is likely to make a difference. We have seen that the processes used to evaluate a doctor's professional competence and conduct are inconsistent, not culturally informed and reinforce structural inequalities prevalent in society. The GMC and NHS Employers and Providers must work together to provide a comprehensive induction programme for all IMGs. This should be complemented by appropriate local induction processes, and appointment of mentors and champions who work with IMGs as they settle into work in the NHS.

\section{Embedding EDI}

We owe it to our new doctors entering our profession doctors to help them develop their own identity while defining and maintaining core values of the NHS as defined in a new 'contract' with our patients and society. Rather than accepting this status quo as inevitable or unchangeable, it is imperative that NHS organisations, wider employers, Universities, HEE take this opportunity to review their processes, Equality, Diversity and Inclusion training and get feedback to ensure their actions are seen as fair and consistent and retain the trust of the profession and the public. The GMC must work with EDI leads, organisations and diaspora medical organisations like BAPIO to introduce cultural sensitivity into understanding domains of Good Medical Practice

\section{Task \& Finish Group}

Finally, we recommend that a Task and Finish Group is set up by GMC to consider how the recommendations can be implemented, how the quality of the interventions can be assured and how their effectiveness can be measured. Working closely with doctors and employers is essential to engage the trust of the medical profession and enable them to provide the best possible care to their patients and the public.

4. Woolf, K., Potts, H. W. W. \& McManus, I. C. Ethnicity and academic performance in UK trained doctors and medical students: systematic review and meta-analysis. BMJ 342, d901 (2011). doi: https://doi.org/10.1136/bmj.d901

5. New data on gender pay gap in medicine. GOV.UK https://www.gov.uk/government/news/new-data-ongender-pay-gap-in-medicine.

6. 1975 - Report of G.M.C. inquiry.pdf. https://www.ncbi.nlm.nih.gov/pmc/articles/PMC167597 $1 /$

7. Good medical practice. https://www.gmc-uk.org/ethicalguidance/ethical-guidance-for-doctors/good-medicalpractice.

8. Dyer, C. Bristol inquiry: Bristol inquiry condemns hospital's "club culture". BMJ 323, 181 (2001). doi: https://doi.org/10.1136/bmj.323.7306.181

9. The Shipman Inquiry. https://assets.publishing.service.gov.uk/government/upl 
oads/system/uploads/attachment_data/file/273227/585 4.pdf

10. Our history. https://www.gmc-uk.org/about/who-weare/our-history.

11. Regulatory_Reform_Consultation_Document.pdf https://assets.publishing.service.gov.uk/government/upl oads/system/uploads/attachment_data/file/655794/Reg ulatory_Reform_Consultation_Document.pdf

12. Health Equity in England: The Marmot Review 10 Years On. The Health Foundation

https://www.health.org.uk/publications/reports/themarmot-review-10-years-on.

13. Black Lives Matter. Wikipedia (2020)

https://en.wikipedia.org/w/index.php?title=Black_Lives_ Matter\&oldid=971334380

14. Chakravorty, I., Bamrah, J. S. \& Mehta, R. A Rainbow Paper - Tackling inequalities, a neo-liberal order in a world after Corona. Sushruta J. Health Policy Opin. 13, (2020). https://doi.org/10.38192/13.3.14

15. NHS Race and Health Observatory. http://www.nhsconfed.org/networks/nhs race and health observatory.

16. Aims of the Observatory.

http://www.nhsconfed.org/networks/nhs race and health observatory/aims of the observatory.

17. Horsfall, S. Doctors who commit suicide while under GMC fitness to practise investigation. 83. https://www.gmcuk.org/-

/media/documents/Internal_review_into_suicide_in_FTP_ processes.pdf_59088696.pdf

18. Walshe, K. \& Offen, N. A very public failure: lessons for quality improvement in healthcare organisations from the Bristol Royal Infirmary. BMJ Qual. Saf. 10, 250-256 (2001). https://doi.org/10.1136/qhc.0100250

19. An update on the GMC's work to address the Francis Recommendations. 11. https://www.gmc-uk.org//media/documents/gmc-update-on-francisrecommendations---final_pdf-53738187.pdf

20. Ham, C. R. Klein, The Politics of the National Health Service, Longman, London, 1983 J. Soc. Policy 13, 359-360 (1984).

21. Archives, T. N. The National Health Service.

https://www.nationalarchives.gov.uk/cabinetpapers/alev elstudies/nhs.htm?WT.ac=The $\% 20$ National $\% 20$ Health $\% 2$ 0Service.

22. CanMEDS Role: Professional : The Royal College of Physicians and Surgeons of Canada.

https://www.royalcollege.ca/rcsite/canmeds/framework /canmeds-role-professional-e

23. Donaldson, L. J. Professional accountability in a changing world. Postgrad. Med.J. 77, 65-67 (2001). https://doi.org/10.1136/pmj.77.904.65

24. Baillie, L. \& Matiti, M. Dignity, equality and diversity: an exploration of how discriminatory behaviour of healthcare workers affects patient dignity. Divers. Equal. Health Care 10, (2013).

https://diversityhealthcare.imedpub.com/abstract/dignit y-equality-and-diversity-an-exploration-of-howdiscriminatory-behaviour-of-healthcare-workers-affectspatient-dignity-1724.html

25. The Bias of 'Professionalism' Standards (SSIR). https://ssir.org/articles/entry/the_bias_of_professionalis m_standards.

26. Powers, B. W., White, A. A., Oriol, N. E. \& Jain, S. H. RaceConscious Professionalism and African American Representation in Academic Medicine. Acad. Med. 91, 913915 (2016). https://doi.org/10.1097/ACM.0000000000001074

27. Weaver, M. What you need to know about the junior doctors' strike. The Guardian (2016). https://www.theguardian.com/society/2016/sep/01/wh at-you-need-to-know-about-the-junior-doctors-strike

28. The Bawa-Garba case | The BMJ. https://www.bmj.com/bawa-garba.
29. Bamrah, J. S. \& Mehta, R. Back to Blame: Sushruta J. Health Policy Opin. 11, 9-12 (2018).

https://doi.org/10.38192/11.1.3

30. Patel F, Chakravorty I. Millennials - The missing piece in the NHS Workforce puzzle? Sushruta 13, (2020). https://doi.org/10.38192/13.1.7

31. Disparities in the risk and outcomes of COVID-19. 92 https://assets.publishing.service.gov.uk/government/upl oads/system/uploads/attachment_data/file/908434/Disp arities_in_the_risk_and_outcomes_of_COVID_August_2020_ update.pdf.

32. Chakravorty, I. et al. An Online Survey of Healthcare Professionals in the COVID-19 Pandemic in the UK: SUSHRUTA J. Health Policy Opin. 13, (2020). https://doi.org/10.38192/13.2.9

33. Wyatt, T. R. et al. 'Whispers and shadows': A critical review of the professional identity literature with respect to minority physicians. Med. Educ. 55, 148-158 (2021).

34. fair-to-refer-report_pdf-79011677.pdf. https://www.gmcuk.org/-/media/documents/fair-to-refer-report_pdf-

79011677.pdf

35. NHS England » Evaluation of the NHS Workforce Race Equality Standard (WRES).

https://www.england.nhs.uk/publication/evaluation-ofthe-nhs-workforce-race-equality-standard-wres/.

36. Participation, E. Equality Act 2010.

https://www.legislation.gov.uk/ukpga/2010/15/contents

37. Unwin, E., Woolf, K., Wadlow, C. \& Dacre, J. Disciplined doctors: Does the sex of a doctor matter? A cross-sectional study examining the association between a doctor's sex and receiving sanctions against their medical registration. BMJ Open 4, e005405 (2014). https://doi.org/10.1136/bmjopen-2014-005405

38. Disciplinary action against physicians: Who is likely to get disciplined? - The American Journal of Medicine. https://www.amjmed.com/article/S00029343(05)00150-6/fulltext

39. The state of medical education and practice in the UK https://www.gmc-uk.org/about/what-we-do-andwhy/data-and-research/the-state-of-medical-educationand-practice-in-the-uk

40. Woolf, K., Rich, A., Viney, R., Needleman, S. \& Griffin, A. Perceived causes of differential attainment in UK postgraduate medical training: a national qualitative study. BMJ Open 6, e013429 (2016)

41. Slowther, A., Lewando Hundt, G. A., Purkis, J. \& Taylor, R. Experiences of non-UK-qualified doctors working within the UK regulatory framework: a qualitative study. J. R. Soc. Med. 105, 157-165 (2012).

42. Majid, A. What lies beneath: getting under the skin of GMC referrals. BMJ 368, (2020). https://doi.org/10.1136/bmj.m338

43. Wise, J. Poor induction and lack of honest feedback is blamed for higher rate of GMC referrals among BAME doctors. BMJ 365, 14391 (2019). https://doi.org/10.1136/bmj.14391

44. Humphrey, C., Hickman, S. \& Gulliford, M. C. Place of medical qualification and outcomes of UK General Medical Council "fitness to practise" process: cohort study. BMJ 342, d1817 (2011). https://doi.org/10.1136/bmj.d1817

45. Rimmer, A. Ethnic minority and non-UK doctors don't think GMC treats all doctors fairly, survey finds. BMJ 348, g3716 (2014). https://doi.org/10.1136/bmj.g3716

46. Fitness to Practise: Review of Serious Misconduct. https://www.gdc-uk.org/docs/defaultsource/research/20170409seriousness-review-finalreport.pdf?sfvrsn=d34194fc_2

47. Review of decision making in our fitness to practise procedures. https://www.gmc-uk.org/about/what-we-doand-why/data-and-research/research-and-insightarchive/review-of-decision-making-in-our-fitness-topractise-procedures 
48. Godlee, F. The GMC, racism, and complaints against doctors. BMJ 312, 1314-1315 (1996). https://doi.org/10.1136/bmj.312.7042.1314

49. Figure 4 - HE student enrolments by personal characteristics 2013/14 to 2017/18 | HESA. https://www.hesa.ac.uk/data-and-analysis/sb252/figure4.

50. Differential attainment in medical education and training | The BMJ. https://www.bmj.com/content/368/bmj.m339.

51. Kmietowicz, Z. Are medical schools turning a blind eye to racism? BMJ 368, m420 (2020). https://doi.org/10.1136/bmj.m420

52. McManus, I. C., Richards, P., Winder, B. C., Sproston, K. A. \& Styles, V. Medical school applicants from ethnic minority groups: identifying if and when they are disadvantaged. BMJ 310, 496-500 (1995). https://doi.org/10.1136/bmj.310.6978.496

53. McManus, I. C., Esmail, A. \& Demetriou, M. Factors affecting likelihood of applicants being offered a place in medical schools in the United Kingdom in 1996 and 1997: retrospective study Commentary: League tables will help Commentary: Some legal aspects arising from the study. BMJ 317, 1111-1117 (1998). https://doi.org/10.1136/bmj.317.7166.1111

54. Linton, S. Taking the difference out of attainment. BMJ 368, (2020). https://doi.org/10.1136/bmj.m438

55. Tackling racial harassment: universities challenged | Equality and Human Rights Commission. https://www.equalityhumanrights.com/en/publicationdownload/tackling-racial-harassment-universitieschallenged.

56. Dyson, M. Caring, supportive, collaborative: a future vision for the NHS. The British Medical Association is the trade union and professional body for doctors in the UK. https://www.bma.org.uk/advice-and-support/nhsdelivery-and-workforce/the-future/caring-supportivecollaborative-a-future-vision-for-the-nhs

57. Racial harassment charter for medical schools. The British Medical Association is the trade union and professional body for doctors in the UK. https://www.bma.org.uk/adviceand-support/discrimination-and-harassment/racialharassment-in-medical-schools/racial-harassmentcharter-for-medical-schools

58. What is revalidation? https://www.gmcuk.org/registration-and-licensing/managing-yourregistration/revalidation/introduction-to-revalidation
59. GMC. Publication and Disclosure Policy. 27.

https://www.gmc-uk.org/-

/media/documents/DC4380_Publication_and_disclosure policy_36609763.pdf

60. How do we check doctors are giving good care? https://www.gmc-uk.org/registration-andlicensing/managing-your-registration/revalidation/howdo-we-check-doctors-are-giving-good-care.

61. Re: Whistleblower was unfairly dismissed in case lasting 12 years, tribunal rules. g2881 (2021). https://www.bmj.com/content/348/bmj.g2881/rr/6959 54

62. Markwell, A. L. \& Wainer, Z. The health and wellbeing of junior doctors: insights from a national survey. Med. J. Aust. 191, 441-444 (2009). https://doi.org/10.5694/j.1326-5377.2009.tb02880.x

63. Gerada, C. Doctors and suicide. Br. J. Gen. Pract. 68, 168169 (2018). https://doi.org/10.3399/bjgp18X695345

64. Suicide in doctors and other health professionals. https://www.practitionerhealth.nhs.uk/suicide-indoctors-and-other-health-prof.

65. Sarafis, P. et al. The impact of occupational stress on nurses' caring behaviors and their health related quality of life. BMC Nurs. 15, 56 (2016). https://doi.org/10.1186/s12912-016-0178-y

66. Tawfik, D. S. et al. Physician Burnout, Well-being, and Work Unit Safety Grades in Relationship to Reported Medical Errors. Mayo Clin. Proc. 93, 1571-1580 (2018). https://doi.org/10.1016/j.mayocp.2018.05.014

67. Bhugra, D. et al. A descriptive study of mental health and wellbeing of doctors and medical students in the UK. Int Rev. Psychiatry 31, 563-568 (2019). https://doi.org/10.1080/09540261.2019.1648621

68. Howe, A. Doctors' health and wellbeing. BMJ 347, f5558 (2013). https://doi.org/10.1136/bmj.f5558

69. New indicators will track doctors' careers to expose racism | The BMJ. https://www.bmj.com/content/370/bmj.m3659.

\section{Acknowledgements}

The authors are grateful to Andrew Timmis and Martin Fischer for their helpful suggestions and editing of this manuscript. 